\title{
Different iron deposition patterns in Parkinson's disease subtypes: a quantitative susceptibility mapping study
}

\author{
Junling Chen ${ }^{1,2 \#}$, Tongtong Cai ${ }^{2 \#}$, Yan $\mathrm{Li}^{1 \#}$, Jieshan Chi ${ }^{1}$, Siming Rong ${ }^{1}$, Chentao He ${ }^{1}$, Xiaohong Li ${ }^{1}$, \\ Piao Zhang ${ }^{1}$, Lijuan Wang ${ }^{1}$, Yuhu Zhang ${ }^{1}$ \\ ${ }^{1}$ Department of Neurology, Guangdong Neuroscience Institute, Guangdong Provincial People's Hospital, Guangdong Academy of Medical Sciences, \\ Guangzhou, China; ${ }^{2}$ Department of Neurology, Shantou Central Hospital, Shantou, China \\ "These authors contributed equally to this work.
}

Correspondence to: Yuhu Zhang. 106 Zhongshan Er Road, Guangzhou 510080, China. Email: yhzhangsd@126.com.

\begin{abstract}
Background: Parkinson's disease (PD) is a heterogeneous neurodegenerative disorder with various subtypes and uncertain pathogenesis. Iron deposition is considered to be involved in the pathological mechanisms of PD. The present study aimed to investigate the iron deposition in deep gray matter in patients with different PD subtypes using quantitative susceptibility mapping (QSM).

Methods: Forty-six PD patients and 22 healthy controls (HCs) were recruited for the study. PD patients were allocated to the tremor-dominant (TD) group $(\mathrm{n}=22)$, postural instability and gait disorder-dominant (PIGD) group ( $\mathrm{n}=19)$, and intermediate group $(\mathrm{n}=5)$. Susceptibility values in deep gray matter nuclei measured by QSM among the PD-TD and PD-PIGD groups and the HCs, as well as the relationship between iron accumulation and clinical motor features, were investigated.

Results: Susceptibility values in the dentate nucleus (DN) were greater in the PD-TD (118.73 \pm 70.45$)$ group than in the PD-PIGD $(72.14 \pm 39.85, \mathrm{P}=0.02)$ group and HCs $(78.26 \pm 41.38, \mathrm{P}=0.042)$. Further, a significant positive correlation was observed between the $\mathrm{DN}$ susceptibility values and tremor scores $(\mathrm{r}=0.324$, $\mathrm{P}=0.028)$. Compared with the HCs $(182.60 \pm 85.35)$, both the PD-TD $(282.00 \pm 102.49, \mathrm{P}=0.006)$ and PDPIGD groups $(284.91 \pm 118.54, \mathrm{P}=0.007)$ exhibited greater susceptibility values in the substantia nigra (SN) pars reticulata. The susceptibility values in the SN pars compacta were also greater in the PD-PIGD group (164.51 \pm 89.44$)$ than in the HCs $(107.78 \pm 63.11, \mathrm{P}=0.048)$.
\end{abstract}

Conclusions: The present study demonstrated various iron deposition patterns in different PD phenotypes. These findings give insight into the pathophysiology underlying different PD phenotypes, and potentially illustrate the involvement of iron deposition in the PD-TD and PD-PIGD subtypes.

Keywords: Iron deposition; Parkinson's disease (PD); quantitative susceptibility mapping (QSM); subtypes

Submitted Feb 15, 2020. Accepted for publication Aug 13, 2020.

doi: 10.21037/qims-20-285

View this article at: http://dx.doi.org/10.21037/qims-20-285

\section{Introduction}

Parkinson's disease (PD) is a common neurodegenerative disorder that manifests with varying combinations of resting tremors, rigidity, bradykinesia, and postural instability. Marked heterogeneity has been demonstrated in the clinical manifestations, disease progression, and prognosis of the disease (1). PD has been increasingly recognized to be a heterogeneous neurodegenerative disorder with various subtypes and underlying neuropathological mechanisms $(2,3)$; therefore, disease-modifying and symptomatic treatment is essential. To date, several methods have been used to categorize PD patients into clinical subtypes based on motor and non-motor features. According to the differences in motor symptoms assessed by the ratio 
of tremor score and postural instability and gait disorder (PIGD) score of the Movement Disorder Society Unified Parkinson's Disease Rating Scale (MDS-UPDRS) (4), PD individuals are subdivided into three subtypes: tremordominant (TD), PIGD-dominant, and the intermediate subtype. These subtypes differ greatly in course, clinical manifestation, disease progression, responsiveness to therapy, and prognosis $(2,5)$. Patients with the PDTD subtype have a slower rate of progression, fewer motor symptoms, and better prognosis than those with the PD-PIGD subtype $(2,6)$. However, the underlying pathophysiology of the clinicopathological heterogeneity in PD remains obscure.

Iron overload has been shown to contribute to the development of some neurodegenerative diseases. Iron homoeostasis is indispensable to normal physiological brain function, because iron participates in oxygen transportation, activation and storage, myelin synthesis, neurotransmitter synthesis, and metabolism $(7,8)$. However, iron dysregulation can potentially cause neuronal degeneration, or even death. Excessive iron catalyzes the oxidation of dopamine, thereby exacerbating the formation of other neurotoxic byproducts (9). The latest findings suggest that iron dysregulation is also closely related to $\alpha$-synuclein and the autophagy-lysosome pathway in PD $(10,11)$. Recent studies have drawn the same conclusion that regional iron levels in the substantia nigra (SN) are greater than those in healthy controls (HCs) (12-14).

Quantitative susceptibility mapping (QSM) is a new, non-invasive technique that quantifies iron deposition, as demonstrated in various studies of the liver and brain, particularly in deep gray matter nuclei, which was confirmed by a post-mortem validation study $(15,16)$. QSM is considered to have higher sensitivity and precision than traditional methods, such as R2* mapping (17). Although QSM has been widely used to study iron accumulation in $\mathrm{PD}$, to the best of our knowledge, no study has reported any differences in iron deposition patterns between the PD-TD and PD-PIGD subtypes using QSM previously. Therefore, in the present study, we employed QSM to systematically investigate the susceptibility difference between the PDTD and PD-PIGD subtypes.

\section{Methods}

\section{Patients}

PD patients and age- and sex-matched HCs were consecutively recruited between April, 2018, and August, 2019, from the Inpatient Department of Guangdong Neuroscience Institute, Guangdong Provincial People's Hospital, China. Informed consent was obtained from all patients. The study was approved by the Ethics Committee of Guangdong Provincial People's Hospital \{No. 2018338H [R1]\} and was performed in accordance with the ethical standards of the 1964 Declaration of Helsinki and its later amendments.

The diagnosis of PD was made according to the MDS clinical diagnostic criteria for PD, devised in 2015 by two specialists with over 10 years of experience in movement disorders (18). For each patient, demographic and clinical data, including sex, age, years of education, duration of illness, and Mini-Mental State Examination (MMSE) score, were collected. The Hoehn and Yahr (H-Y) scale and MDSUPDRS III were used to assess the severity of PD by a trained physician. Patients were requested not to take antiparkinsonian drugs for at least 12 hours overnight before the assessments were conducted.

PD patients were allocated to the PD-TD group, PDPIGD group, or intermediate group according to the ratio of the mean tremor score to the mean PIGD score, as assessed by the MDS-UPDRS (MDS-UPDRS TD/ PIGD ratio). Based on a previously published method, we set the TD/PIGD ratio at $\geq 1.15$ for TD classification, $\leq 0.9$ for PIGD classification, and ratio $>0.9$ and $<1.15$ for intermediate classification (4). Patients with a PIGD score of 0 were allocated to the PD-TD group, whereas patients with a TD score of 0 were allocated to the PD-PIGD group.

The exclusion criteria were as follows: (I) a history of cerebrovascular accidents, brain tumor, or other neurological disorders; (II) with severe internal or surgical diseases; (III) a history of severe anxiety, depression, schizophrenia, or another psychosocial illness; or (IV) unwilling or unable to undergo magnetic resonance imaging (MRI) examination, or contraindications for MRI examination.

None of the HCs reported a history of neurological or psychiatric disorders, or any current medical problems.

\section{Image protocol and postprocessing}

All patients were scanned in a 3.0 T MR machine (Signa Excite, GE Medical System) with an 8-channel, phasedarray head coil. Standard T1- and T2-weighted images were obtained for structural observation. Meanwhile, 3D-enhanced gradient echo T2 star-weighted angiography 

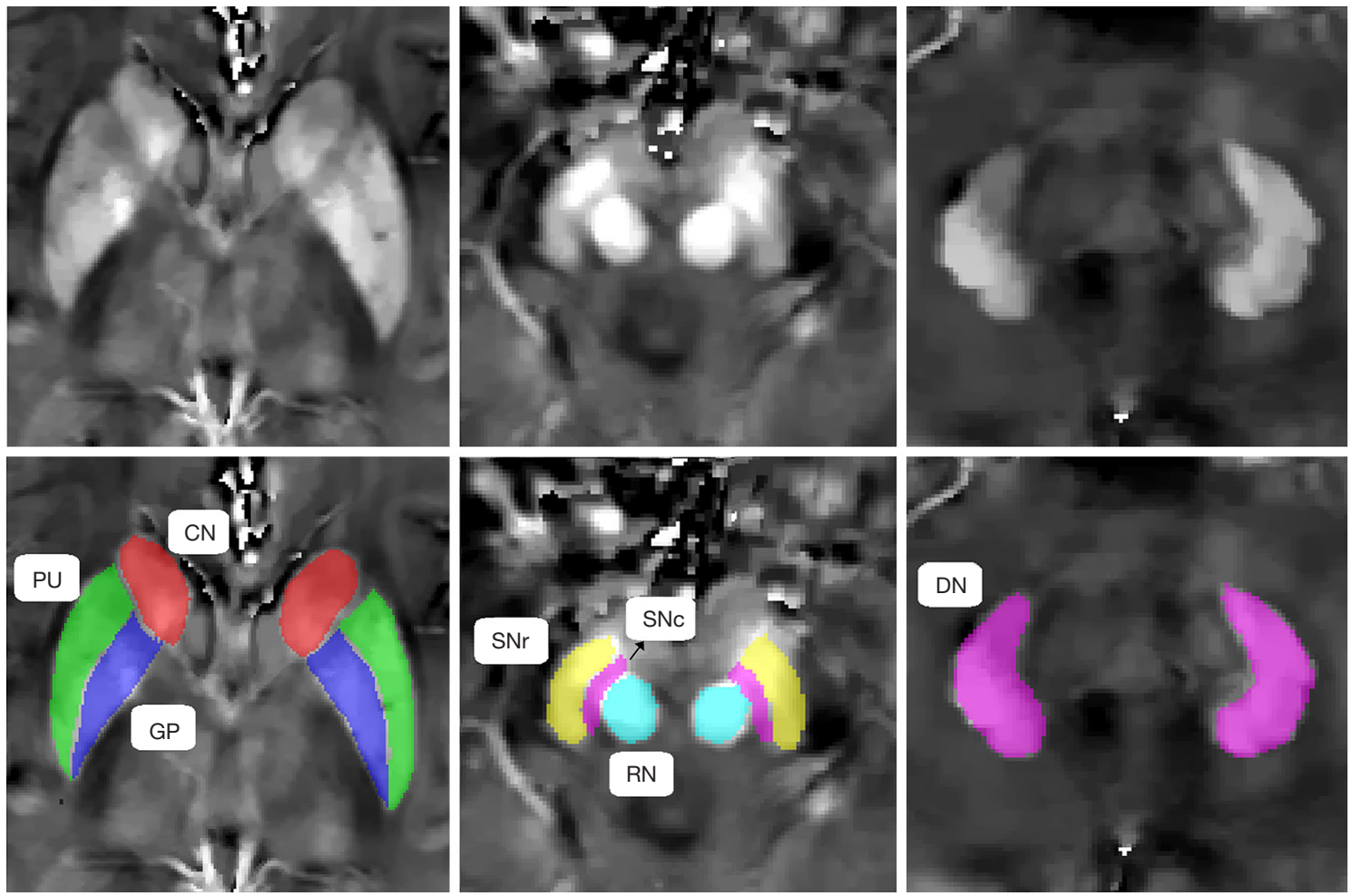

Figure 1 Representative quantitative susceptibility mapping (QSM) images of the deep gray matter nuclei of a Parkinson's disease (PD) patient. CN, caudate nucleus; DN, dentate nucleus; GP, globus pallidus; PU, putamen; RN, red nucleus; SNc, substantia nigra pars compacta; SNr, substantia nigra pars reticulata.

sequences were acquired with 13 spaced echoes (repetition time $/$ echo time $=89.2 / 2.6-36.4 \mathrm{~ms}$, flip angle $=30^{\circ}$, field of view $=240 \mathrm{~mm} \times 240 \mathrm{~mm}$, slice thickness $=3 \mathrm{~mm}$, number of slices $=72$, voxel size $=0.75 \times 1.07 \times 3 \mathrm{~mm}^{3}$, bandwidth $=62.5 \mathrm{~Hz} /$ pixel, matrix size $=320 \times 224)$. QSM reconstruction was performed in MATLAB (version R2016b; MathWorks) using the morphology enabled dipole inversion (MEDI) algorithm (lambda $=900$, lambda $\mathrm{CSF}=10$, merit $=1, \mathrm{cg}_{-}$ max_iter $=100$, cg_tol $=0.01$, max_iter $=10$, tol_norm_ratio $=0.1$ ), according to previously published studies (19-22). Briefly, the QSM image was obtained by unwrapping the phase image, removing the background field, and reconstructing the image through the MEDI algorithm.

\section{Regions of interest (ROI) analysis}

ITK-SNAP3.6.0 (www.itksnap.org) was used to perform the manual segmentation and measure the magnetic susceptibility, as described in a previously published study (23). Data for each region were obtained from the maximal visible slice. An experienced neuroradiologist, who was blinded to the patients' information, was responsible for the ROI analysis. We selected the bilateral globus pallidus (GP), putamen (PU), caudate nucleus $(\mathrm{CN}), \mathrm{SN}$ pars reticulata (SNr), SN pars compacta (SNc), red nucleus $(\mathrm{RN})$, and dentate nucleus (DN) as the ROIs, and the sum of the susceptibility values of the bilateral regions was calculated. To investigate the reproducibility and reliability of the QSM data, the same experienced neuroradiologist responsible for the ROI analysis remeasured the nuclei 3 months later. Representative QSM images of the deep gray matter nuclei of a PD patient are shown in Figure 1.

\section{Statistical analysis}

Statistical analysis was performed using IBM SPSS Statistics version 19.0 (Armonk, NY, IBM Corp). The KolmogorovSmirnov test was used to analyze the normality of the data. Demographic and clinical data are presented as mean \pm standard deviation (SD) and were compared between groups by using the $\chi^{2}$-test for sex; two-tailed Student's $t$-test, one-way analysis of variance (ANOVA), Mann-Whitney 
Table 1 Patients' demographic and clinical data

\begin{tabular}{|c|c|c|c|c|c|}
\hline Variable & PD-TD (n=22) & PD-PIGD (n=19) & HCs $(n=22)$ & Statistics $\left(\chi^{2} / F / Z / t\right)$ & $P$ value \\
\hline Age at visit (years) ${ }^{\ddagger}$ & $61.14 \pm 8.75$ & $62.47 \pm 7.07$ & $59.59 \pm 9.40$ & 0.587 & 0.056 \\
\hline Education $\left(\right.$ years) $^{\ddagger}$ & $9.48 \pm 4.15$ & $9.16 \pm 5.43$ & $9.55 \pm 4.94$ & 0.037 & 0.096 \\
\hline MMSE§ & $26.91 \pm 2.88$ & $26.42 \pm 5.00$ & $28.36 \pm 1.65$ & 3.727 & 0.155 \\
\hline $\mathrm{H}-\mathrm{Y}$ scale" & $2.11 \pm 0.58$ & $2.58 \pm 0.67$ & & -2.123 & $0.034^{*}$ \\
\hline Tremor score" & $7.18 \pm 2.92$ & $1.53 \pm 2.53$ & & -4.632 & $0.00^{* *}$ \\
\hline PIGD score" & $2.32 \pm 1.96$ & $3.90 \pm 2.71$ & & -2.197 & $0.028^{*}$ \\
\hline MDS-UPDRS $\mathrm{II}^{\dagger \dagger}$ & $36.30 \pm 15.95$ & $37.74 \pm 14.64$ & & -0.3 & 0.766 \\
\hline
\end{tabular}

${ }^{*}, \mathrm{P}<0.05 ;{ }^{*}, \mathrm{P}<0.01 .{ }^{\dagger}, \mathrm{P}$ value for sex distribution in the three groups was calculated using $\chi^{2}$-test; ${ }^{\ddagger}, \mathrm{P}$ value was calculated using one-way analysis of variance; ${ }^{\S}$, P value was calculated using Kruskal-Wallis $\mathrm{H}$-test; ", $\mathrm{P}$ value was calculated using Mann-Whitney U-test; ${ }^{\dagger \dagger}$, $\mathrm{P}$ value was calculated using two-tailed Student's $t$-test. HCs, healthy controls; H-Y scale, Hoehn and Yahr scale; MDS-UPDRS III, Movement Disorder Society Unified Parkinson's Disease Rating Scale part III; MMSE, Mini-Mental State Examination; PD, Parkinson's disease; PIGD, postural instability and gait disorder; TD, tremor dominant.

$U$-test, or Kruskal-Wallis H-test were used for continuous variables, as appropriate, according to the normal distribution type and the homogeneity of variance of the data. ANOVA was also used to evaluate the susceptibility values between the PD-TD and PD-PIGD groups and HCs. Post hoc tests with a Bonferroni correction for multiple comparisons were performed to reduce type I errors for the results. $\mathrm{P}<0.05$ was considered statistically significant. The relationship between susceptibility values and clinical motor features (e.g., MDS-UPDRS III score, H-Y scale, tremor score, and PIGD score) was analyzed by partial correlation analysis, with age, sex, and the MMSE score as covariates.

\section{Results}

\section{Participants' demographics and clinical status}

A total of 46 patients with PD fulfilled our study criteria and were allocated to 3 groups: the PD-TD group ( $\mathrm{n}=22$, $47.8 \%$ ), PD-PIGD group ( $\mathrm{n}=19,41.3 \%)$, and intermediate group $(\mathrm{n}=5,10.9 \%)$. The intermediate group manifested without symptom preference and had a small sample size; consequently, it was excluded from the intergroup QSM value comparisons (3). For data continuity, we incorporated all participants, including those in the intermediate group, into the correlation analysis to evaluate the relationship between iron deposition and clinical motor features. Twenty-two HCs matched for age and sex were also included in the study. The demographic and clinical features of the patients are summarized in Table 1 . There were no significant differences in age, sex, years of education, or MMSE score between the participants $(\mathrm{P}>0.05)$. The PDTD and PD-PIGD groups had similar disease duration and MDS-UPDRS III score $(\mathrm{P}>0.05)$; however, the PDTD subtype had greater tremor scores $(\mathrm{P}<0.01)$ and a lower PIGD score $(\mathrm{P}=0.037)$ and $\mathrm{H}-\mathrm{Y}$ scale score $(\mathrm{P}=0.02)$, consistent with the clinical features of each subtype.

\section{ROI analysis}

ANOVA revealed different iron deposition patterns among the PD-TD and PD-PIGD group and HCs in the DN, as well as in the SN. In the DN region, the susceptibility values were significantly greater in the $\mathrm{PD}-\mathrm{TD}$ group than in the PD-PIGD group ( $\mathrm{P}=0.02)$ and HCs $(\mathrm{P}=0.042)$, whereas the PD-PIGD group showed a non-significant difference with the HCs $(\mathrm{P}=1.00)$ (Figure 2). Compared with the HCs, the PD-TD $(\mathrm{P}=0.006)$ and $\mathrm{PD}-\mathrm{PIGD}$ groups $(\mathrm{P}=0.007)$ exhibited greater susceptibility values in the $\mathrm{SNr}$ (Figure 2). Further, the PD-PIGD group showed greater susceptibility compared to the HCs in the SNc region. No significant difference was found in the GP $(\mathrm{P}=0.207)$, $\mathrm{PU}(\mathrm{P}=0.968), \mathrm{CN}(\mathrm{P}=0.195)$, or $\mathrm{RN}(\mathrm{P}=0.321)$ regions. A significant positive correlation $(\mathrm{r}=0.324, \mathrm{P}=0.028)$ was also observed between the susceptibility values of the $\mathrm{DN}$ and the tremor scores in all PD patients, including the intermediate group (Figure 3). No significant correlations 


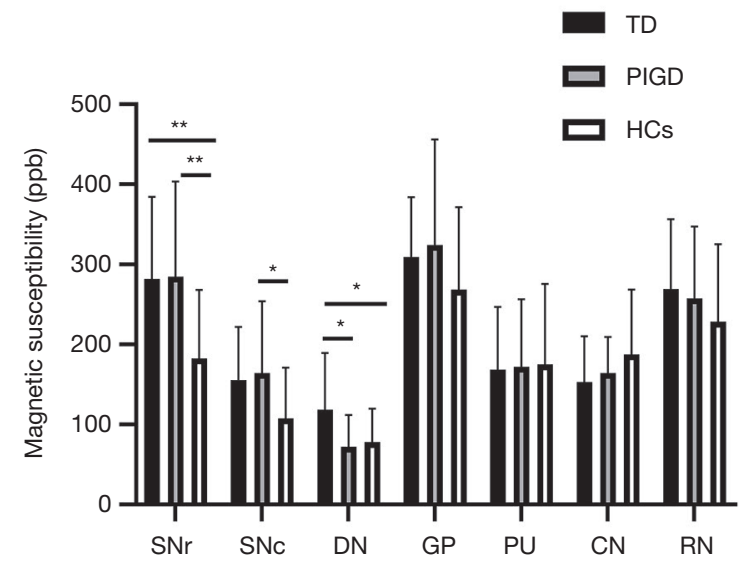

Figure 2 Comparison of susceptibility values in deep gray matter nuclei between the tremor-dominant (TD) and postural instability and gait disorder (PIGD) groups and healthy controls (HCs). **, $\mathrm{P}<0.01$; * $\mathrm{P}<0.05$. CN, caudate nucleus; $\mathrm{DN}$, dentate nucleus; GP, globus pallidus; PU, putamen; RN, red nucleus; SNc, substantia nigra pars compacta; $\mathrm{SNr}$, substantia nigra pars reticulata.

were found between the susceptibility values of other nuclei and clinical features.

\section{Discussion}

In the current study, we employed QSM to investigate regional differences in iron deposition between TD and PIGD PD patients and HCs. The most important discovery was the variability in the iron level among the groups in the $\mathrm{DN}$ region, which presented as the $\mathrm{DN}$ of the $\mathrm{PD}-\mathrm{TD}$ group having significantly greater susceptibility values than the DN of the PD-PIGD group and HCs. Furthermore, the patients with the PD-PIGD subtype had increased iron deposition in both the $\mathrm{SNr}$ and $\mathrm{SNc}$ compared with the HCs. Taken together, these findings revealed different iron distribution patterns between the PD subtypes, thus giving important insights into the pathophysiological mechanism underlying the PD phenotype.

In accordance with previously published studies, we found that the susceptibility values were significantly greater in the DN of the PD-TD group than in that of the HCs $(3,24)$. Additionally, the susceptibility values in the DN were positively correlated with tremor severity in PD patients. Our observations indicated that parkinsonian tremors may be associated with iron deposition in the $\mathrm{DN}$, which is the largest nucleus of the cerebello-thalamocortical (CTC) circuit. The dysfunction of the basal ganglia

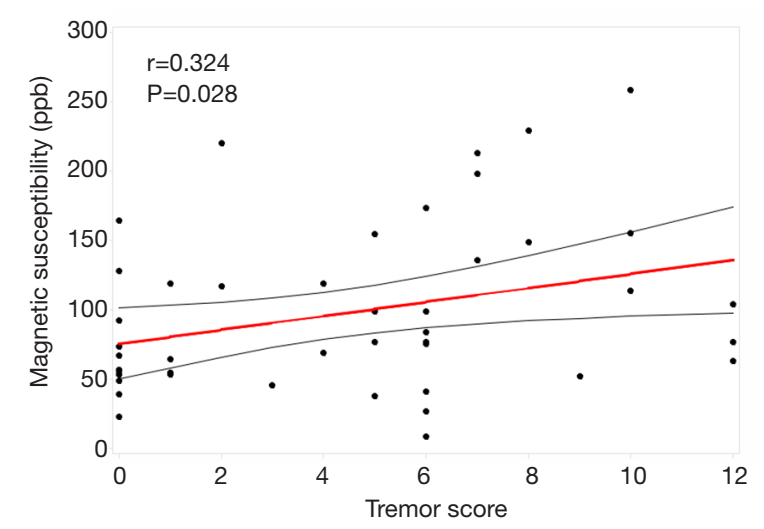

Figure 3 Relationship between the susceptibility values in the dentate nucleus and the tremor score.

(BG), triggered by $\mathrm{SN}$ degeneration, is considered to play a key role in the generation of motor impairment through the striatal- thalamo-cortical circuit $(25,26)$. However, this mechanism still fails to fully explain the pathophysiology of tremors in PD. In their review of data from postmortem and nuclear imaging studies, Helmich et al. postulated that tremors were an independent symptom in PD, and the PDTD subtype patients had milder cell loss and dopaminergic dysfunction than non-tremor PD patients (27). The recent discovery of a link between the cerebellum and BG, which forms an integrated functional network (28), has aroused interest in the role of the cerebellum in PD. According to the dimmer-switch model, tremor episodes are triggered by the BG, but tremor intensity is modulated in the CTC circuit (27). Using a resting-state functional MRI (fMRI) study, Chen et al. found that PD-TD patients had greater low-frequency fluctuation values in the right cerebellum than both the PD-PIGD patients and HCs, which supports the notion that hyperactivity in the cerebellum is an underlying feature in the pathophysiology of parkinsonian tremors (29). In keeping with these findings, our results supported the assumption that dysfunction of the CTC circuit contributes to tremors. Furthermore, resting tremors in PD usually show strong interindividual variation in the therapeutic response to dopaminergic medication. Using combined electromyography-fMRI, researchers have reported that dopamine-resistant tremor patients showed increased tremor-related activity in non-dopaminergic areas (e.g., the cerebellum) (30). Combined with our results, these findings indicate that other potential treatments for dopamine-resistant PD tremors, such as iron chelators, are worth investigating. 
Regarding parkinsonian gait and postural disturbance, we found that the DN of the PD-PIGD group had significantly lower susceptibility values than the DN of the PD-TD group. Considering that the cerebellum is vital to ongoing movement regulation and stable posture maintenance, ours is an important finding that, to the best of our knowledge, has not been reported before. A previous study that used QSM to evaluate the whole-brain pattern of magnetic susceptibility perturbations in PD reported a significant QSM reduction in the cerebellar DN, which can possibly be explained by the fact that the patient cohort recruited was predominantly non-tremor-dominant PD (31). In a previously published QSM study on the $\mathrm{DN}, \mathrm{He}$ et al. found that QSM values in the $\mathrm{DN}$ were increased in the PD-TD group, but tended to be decreased in the PD-AR group, which suggested that there was a dependence of the motor phenotype on iron deposition (3). Furthermore, Chen et al. also postulated that hypoactivity in the cerebellum in the PD-PIGD subtype, opposite to that in the PD-TD subtype, may be involved in the gait/postural disturbance in PD (29). Moreover, although the consequence of iron overload was the focus of our investigation, the potential harm of iron deficiency cannot be ignored (7). Taken together, our results indicate that alterations in iron levels in the DN may be an underlying feature in the pathophysiology of $\mathrm{PD}$ motor phenotypes.

Another finding of the present study was that the PDTD and PD-PIGD groups showed greater susceptibility values in the SNr than the HCs. Further, the PD-PIGD group showed greater susceptibility compared to the HCs in the SNc. It is generally accepted that dopaminergic neuron death and the reduction of neuromelanin (NM) mainly occur in the $\mathrm{SNc}$, and it has been reported that this is accompanied by the deposition of iron (14). A previously published study found that there are some inherent effects of SN iron deposition on PD clinical symptoms (32). Jin et al. suggested that nigral iron deposition, which was correlated with decreased serum ceruloplasmin levels, is a risk factor for PD across multiple motor phenotypic expressions (33). Our finding was consistent with previous neuropathological findings, which demonstrated more severe SNc neuronal loss and faster progression among PD-PIGD patients. However, we did not observe an iron content increase in the pars compacta in the PDTD subtype when compared to the HCs, which was an unexpected finding. One limitation of our work was the relatively low spatial resolution, which could explain the unexpected outcome. Precise ROI localization in the
QSM was also challenging with manual segmentation. Nigrosomes, distributing non-uniformly throughout the $\mathrm{SNc}$, are rich in NM. Melanin binds to a number of metal ions, of which iron ions are the most abundant (34). Of note, nigrosome 1 (N1) is considered to be the largest nigrosome in the SNc. Therefore, we can assume that the measurement of susceptibility value in the N1 can reflect the iron level of the $\mathrm{SN}$ in $\mathrm{PD}$ more precisely. In a recent study, Cheng et al. reported that the N1 sign can be consistently visualized using true susceptibility-weighted images with a resolution of at least $0.67 \times 0.67 \times 1.34 \mathrm{~mm}^{3}(35)$. We aim to adopt atlas-based auto-segmentation for deep gray matter nuclei or novel and high-quality positioning technology in our future research.

No statistically significant difference was observed in the $\mathrm{SNr}$ or SNc between the two PD groups. This could be because dopaminergic neuron death in the $\mathrm{SN}$ is a common pathological feature of PD, regardless of the subtype (PDTD or PD-PIGD), which makes it helpful for diagnosing $\mathrm{PD}$, but not in differentiating between PD subtypes. Some have argued that the PD-TD subtype can transform into the PD-PIGD subtype with disease progression, indicating that these subtypes could be the equivalent of different stages of PD, rather than distinct biologic entities (1). Therefore, the final outcome of the susceptibility values may not be representative, as most PD-TD patients enrolled in our study had accompanying PIGD symptoms. In subsequent research, we may recruit PD-TD patients who manifest without PIGD symptoms to further investigate the susceptibility values in the $\mathrm{SN}$ between the two subtypes. Besides, the small number of recruited patients may have been a limitation of the present study.

Considering the previous discovery that dopaminergic neuron loss and death in the GP were different among the PD subtypes (36), we also compared the susceptibility values in the GP. In contrast to the $\mathrm{SN}$ and DN, only a nonsignificant susceptibility increment was observed in the GP of the PD patients in our study. However, studies on pallidal iron content are conflicting, which may be explained by spatial and temporal dimensions. It is easier to obtain a negative result when quantifying the susceptibility of the entire GP, as iron content decreases in the pars interna and increases in the pars externa in PD. Furthermore, pallidal iron loading may not be obvious in the early stage of PD (31). Another reason that should not be discounted is the relatively smaller sample size of the PD-PIGD group in the present study. Future research is required to confirm whether iron deposition in the GP is different in the PD 
phenotype.

There were several limitations in the present study. First, the small sample size may lead to uncertainty in the results of the analysis; therefore, large cohorts are needed to verify the outcome. Additionally, manual segmentation of the ROIs may affect the accuracy of the results to some extent. Atlas-based auto-segmentation for the deep gray matter nuclei or novel positioning technology should be considered in future research. Further limitations related to disadvantages of the imaging technique, such as the relatively low spatial resolution. High-quality image technology, such as 7.0T MRI, is necessary to investigate small structures in the future. Additionally, as the PD-TD subtype may develop into the PD-PIGD subtype during the processing of $\mathrm{PD}$, a longitudinal study is warranted.

\section{Conclusions}

Our findings suggest that there are different patterns of iron deposition in the PD-TD and PD-PIGD subtypes. In contrast to the PD-PIGD subtype and the HCs, the iron content was found to be greater in the $\mathrm{DN}$ in the PD-TD subtype. The PD-PIGD subtype showed greater iron deposition in both the $\mathrm{SNr}$ and $\mathrm{SNc}$. These findings suggest that iron dysregulation may contribute to the pathophysiology of the PD phenotype. Longitudinal studies are needed to confirm our results.

\section{Acknowledgments}

Funding: This work was supported by Guangzhou Municipal People's Livelihood Science and Technology Project (No. 201803010085); Key Program of Natural Science Foundation of Guangdong Province, China (No. 2017B030311015); High-level Hospital Construction Project (DFJH201907); Supporting Research Funds for Outstanding Young Medical Talents in Guangdong Province (KJ012019442); and National Key R\&D Program of China (No. 2017YFC1310200). The authors thank Dr. Chunling Liu (Imaging Department, Guangdong Provincial People's Hospital) for her support in the image collection and processing. The authors also thank all of the study participants.

\section{Footnote}

Conflicts of Interest: All authors have completed the ICMJE uniform disclosure form (available at http://dx.doi. org/10.21037/qims-20-285). The authors have no conflicts of interest to declare.

Ethical Statement: The study was approved by the Ethics Committee of Guangdong Provincial People's Hospital \{No. $2018338 \mathrm{H}$ [R1]\} and was performed in accordance with the ethical standards of the 1964 Declaration of Helsinki and its later amendments. Informed consent was obtained from all patients.

Open Access Statement: This is an Open Access article distributed in accordance with the Creative Commons Attribution-NonCommercial-NoDerivs 4.0 International License (CC BY-NC-ND 4.0), which permits the noncommercial replication and distribution of the article with the strict proviso that no changes or edits are made and the original work is properly cited (including links to both the formal publication through the relevant DOI and the license). See: https://creativecommons.org/licenses/by-nc-nd/4.0/.

\section{References}

1. Nutt JG. Motor subtype in Parkinson's disease: Different disorders or different stages of disease? Mov Disord 2016;31:957-61.

2. Thenganatt MA, Jankovic J. Parkinson disease subtypes. JAMA Neurol 2014;71:499-504.

3. He N, Huang P, Ling H, Langley J, Liu C, Ding B, Huang J, Xu H, Zhang Y, Zhang Z, Hu X, Chen S, Yan F. Dentate nucleus iron deposition is a potential biomarker for tremor-dominant Parkinson's disease. NMR Biomed 2017;30:10.1002/nbm.3554.

4. Stebbins GT, Goetz CG, Burn DJ, Jankovic J, Khoo TK, Tilley BC. How to identify tremor dominant and postural instability/gait difficulty groups with the movement disorder society unified Parkinson's disease rating scale: comparison with the unified Parkinson's disease rating scale. Mov Disord 2013;28:668-70.

5. Kalia LV, Lang AE. Parkinson's disease. Lancet 2015;386:896-912.

6. Konno T, Deutschlander A, Heckman MG, Ossi M, Vargas ER, Strongosky AJ, van Gerpen JA, Uitti RJ, Ross OA, Wszolek ZK. Comparison of clinical features among Parkinson's disease subtypes: A large retrospective study in a single center. J Neurol Sci 2018;386:39-45.

7. Sian-Hülsmann J, Mandel S, Youdim MB, Riederer P. The relevance of iron in the pathogenesis of Parkinson's disease. J Neurochem 2011;118:939-57. 
8. Ward RJ, Zucca FA, Duyn JH, Crichton RR, Zecca L. The role of iron in brain ageing and neurodegenerative disorders. Lancet Neurol 2014;13:1045-60.

9. Hare DJ, Double KL. Iron and dopamine: a toxic couple. Brain 2016;139:1026-35.

10. Chen B, Wen X, Jiang H, Wang J, Song N, Xie J. Interactions between iron and $\alpha$-synuclein pathology in Parkinson's disease. Free Radic Biol Med 2019;141:253-60.

11. Chen LL, Huang YJ, Cui JT, Song N, Xie J. Iron Dysregulation in Parkinson's Disease: Focused on the Autophagy-Lysosome Pathway. ACS Chem Neurosci 2019;10:863-71.

12. Ghassaban K, He N, Sethi SK, Huang P, Chen S, Yan F, Haacke EM. Regional High Iron in the Substantia Nigra Differentiates Parkinson's Disease Patients From Healthy Controls. Front Aging Neurosci 2019;11:106.

13. Chen Q, Chen Y, Zhang Y, Wang F, Yu H, Zhang C, Jiang Z, Luo W. Iron deposition in Parkinson's disease by quantitative susceptibility mapping. BMC Neurosci 2019;20:23.

14. Langley J, Huddleston DE, Sedlacik J, Boelmans K, Hu XP. Parkinson's disease-related increase of 'T2*-weighted hypointensity in substantia nigra pars compacta. Mov Disord 2017;32:441-9.

15. Liu S, Wang C, Zhang X, Zuo P, Hu J, Haacke EM, Ni $H$. Quantification of liver iron concentration using the apparent susceptibility of hepatic vessels. Quant Imaging Med Surg 2018;8:123-34.

16. Langkammer C, Schweser F, Krebs N, Deistung A, Goessler W, Scheurer E, Sommer K, Reishofer G, Yen K, Fazekas F, Ropele S, Reichenbach JR. Quantitative susceptibility mapping (QSM) as a means to measure brain iron? A post mortem validation study. Neuroimage 2012;62:1593-9.

17. Li G, Zhai G, Zhao X, An H, Spincemaille P, Gillen KM, Ku Y, Wang Y, Huang D, Li J. 3D texture analyses within the substantia nigra of Parkinson's disease patients on quantitative susceptibility maps and R2* maps. NeuroImage 2019;188:465-72.

18. Postuma RB, Berg D, Stern M, Poewe W, Olanow CW, Oertel W, Obeso J, Marek K, Litvan I, Lang AE, Halliday G, Goetz CG, Gasser T, Dubois B, Chan P, Bloem BR, Adler CH, Deuschl G. MDS clinical diagnostic criteria for Parkinson's disease. Mov Disord 2015;30:1591-601.

19. Wang Y, Liu T. Quantitative susceptibility mapping (QSM): Decoding MRI data for a tissue magnetic biomarker. Magn Reson Med 2015;73:82-101.

20. Kressler B, de Rochefort L, Liu T, Spincemaille P, Jiang Q,
Wang Y. Nonlinear regularization for per voxel estimation of magnetic susceptibility distributions from MRI field maps. IEEE Trans Med Imaging 2010;29:273-81.

21. de Rochefort L, Brown R, Prince MR, Wang Y. Quantitative MR susceptibility mapping using piecewise constant regularized inversion of the magnetic field. Magn Reson Med 2008;60:1003-9.

22. Zhou D, Liu T, Spincemaille P, Wang Y. Background field removal by solving the Laplacian boundary value problem. NMR Biomed 2014;27:312-9.

23. Guan X, Xuan M, Gu Q, Huang P, Liu C, Wang N, Xu $\mathrm{X}$, Luo W, Zhang M. Regionally progressive accumulation of iron in Parkinson's disease as measured by quantitative susceptibility mapping. NMR Biomed 2017;30:10.1002/ nbm.3489.

24. Guan X, Xuan M, Gu Q, Xu X, Huang P, Wang N, Shen Z, $\mathrm{Xu}$ J, Luo W, Zhang M. Influence of regional iron on the motor impairments of Parkinson's disease: A quantitative susceptibility mapping study. J Magn Reson Imaging 2017;45:1335-42.

25. Hou Y, Ou R, Yang J, Song W, Gong Q, Shang H. Patterns of striatal and cerebellar functional connectivity in early-stage drug-naïve patients with Parkinson's disease subtypes. Neuroradiology 2018;60:1323-33.

26. Wu T, Hallett $M$. The cerebellum in Parkinson's disease. Brain 2013;136:696-709.

27. Helmich RC, Hallett M, Deuschl G, Toni I, Bloem BR. Cerebral causes and consequences of parkinsonian resting tremor: a tale of two circuits? Brain 2012;135:3206-26.

28. Bostan AC, Dum RP, Strick PL. The basal ganglia communicate with the cerebellum. Proc Natl Acad Sci U S A 2010;107:8452-6.

29. Chen HM, Wang ZJ, Fang JP, Gao LY, Ma LY, Wu T, Hou YN, Zhang JR, Feng T. Different Patterns of Spontaneous Brain Activity between Tremor-Dominant and Postural Instability/Gait Difficulty Subtypes of Parkinson's Disease: A Resting-State fMRI Study. CNS Neurosci Ther 2015;21:855-66.

30. Dirkx MF, Zach H, van Nuland A, Bloem BR, Toni I, Helmich RC. Cerebral differences between dopamineresistant and dopamine-responsive Parkinson's tremor. Brain 2019;142:3144-57.

31. Acosta-Cabronero J, Cardenas-Blanco A, Betts MJ, Butryn M, Valdes-Herrera JP, Galazky I, Nestor PJ. The wholebrain pattern of magnetic susceptibility perturbations in Parkinson's disease. Brain 2017;140:118-31.

32. An H, Zeng X, Niu T, Li G, Yang J, Zheng L, Zhou W, Liu H, Zhang M, Huang D, Li J. Quantifying iron 
deposition within the substantia nigra of Parkinson's disease by quantitative susceptibility mapping. J Neurol Sci 2018;386:46-52.

33. Jin L, Wang J, Jin H, Fei G, Zhang Y, Chen W, Zhao L, Zhao N, Sun X, Zeng M, Zhong C. Nigral iron deposition occurs across motor phenotypes of Parkinson's disease. Eur J Neurol 2012;19:969-76.

34. Zecca L, Stroppolo A, Gatti A, Tampellini D, Toscani M, Gallorini M, Giaveri G, Arosio P, Santambrogio P, Fariello RG, Karatekin E, Kleinman MH, Turro N, Hornykiewicz O, Zucca FA. The role of iron and copper molecules in the neuronal vulnerability of locus coeruleus

Cite this article as: Chen J, Cai T, Li Y, Chi J, Rong S, He C, Li X, Zhang P, Wang L, Zhang Y. Different iron deposition patterns in Parkinson's disease subtypes: a quantitative susceptibility mapping study. Quant Imaging Med Surg 2020;10(11):2168-2176. doi: 10.21037/qims-20-285 and substantia nigra during aging. Proc Natl Acad Sci U S A 2004;101:9843-8.

35. Cheng Z, He N, Huang P, Li Y, Tang R, Sethi SK, Ghassaban K, Yerramsetty KK, Palutla VK, Chen S, Yan F, Haacke EM. Imaging the Nigrosome 1 in the substantia nigra using susceptibility weighted imaging and quantitative susceptibility mapping: An application to Parkinson's disease. Neuroimage Clin 2020;25:102103.

36. Rajput AH, Sitte HH, Rajput A, Fenton ME, Pifl C, Hornykiewicz O. Globus pallidus dopamine and Parkinson motor subtypes: clinical and brain biochemical correlation. Neurology 2008;70:1403-10. 